\title{
Spectroscopic Mass and Host-star Metallicity Measurements for Newly Discovered Microlensing Planet OGLE-2018-BLG-0740Lb
}

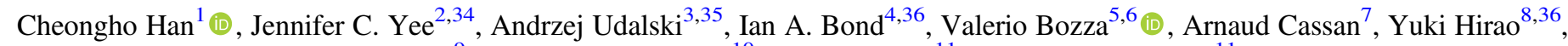
Subo Dong ${ }^{9}$ (i), Juna A. Kollmeier ${ }^{10}$, Nidia Morrell ${ }^{11}$, Konstantina Boutsia ${ }^{11}$ (i)

(Leading authors),

Michael D. Albrow ${ }^{12}$ (1) , Sun-Ju Chung ${ }^{13,14}$ (1) , Andrew Gould ${ }^{13,15,16}$, Kyu-Ha Hwang ${ }^{13}$ (1) , Chung-Uk Lee $^{13}$, Yoon-Hyun Ryu ${ }^{13}$, In-Gu Shin ${ }^{13}$, Yossi Shvartzvald ${ }^{17}$, Youn Kil Jung ${ }^{13}$, Doeon Kim ${ }^{1}$, Woong-Tae Kim ${ }^{18}$, Sang-Mok Cha ${ }^{13,19}$, Dong-Jin Kim ${ }^{13}$, Hyoun-Woo Kim ${ }^{13}$, Kyeongsoo Hong ${ }^{1}$ (1), Seung-Lee Kim ${ }^{13,14}$, Dong-Joo Lee ${ }^{13}$, Yongseok Lee ${ }^{13,19}$, Byeong-Gon Park ${ }^{13,14}$, Richard W. Pogge ${ }^{15}$, Weicheng Zang ${ }^{20}$

(The KMTNet Collaboration),

Przemek Mróz ${ }^{3}$, Michał K. Szymański ${ }^{3}$, Jan Skowron ${ }^{3}$ (1) Radek Poleski $^{15}$, Igor Soszyński ${ }^{3}$, Paweł Pietrukowicz ${ }^{3}$ (1), Szymon Kozłowski ${ }^{3}$, Krzysztof Ulaczyk ${ }^{21}$ (D), Krzysztof A. Rybicki ${ }^{3}$, Patryk Iwanek ${ }^{3}$ (1) , Marcin Wrona ${ }^{3}$

(The OGLE Collaboration),

and

Fumio Abe ${ }^{22}$, Richard Barry ${ }^{23}$, David P. Bennett ${ }^{23,24}$ (10), Aparna Bhattacharya ${ }^{23,24}$, Martin Donachie ${ }^{25}$, Akihiko Fukui ${ }^{26,27}$ (10), Yoshitaka Itow $^{22}$ (1), Kohei Kawasaki ${ }^{8}$, Iona Kondo ${ }^{8}$, Naoki Koshimoto ${ }^{28,29}$ (1), Man Cheung Alex Li ${ }^{25}$, Yutaka Matsubara ${ }^{22}$, Yasushi Muraki $^{22}$, Shota Miyazaki ${ }^{8}$ (1), Masayuki Nagakane ${ }^{8}$, Clément Ranc ${ }^{23}$, Nicholas J. Rattenbury ${ }^{25}$ (1) , Haruno Suematsu ${ }^{8}$, Denis J. Sullivan ${ }^{30}$, Takahiro Sumi ${ }^{8}$, Daisuke Suzuki ${ }^{31}$ (1) , Paul J. Tristram ${ }^{32}$, and Atsunori Yonehara ${ }^{33}$

(The MOA Collaboration)

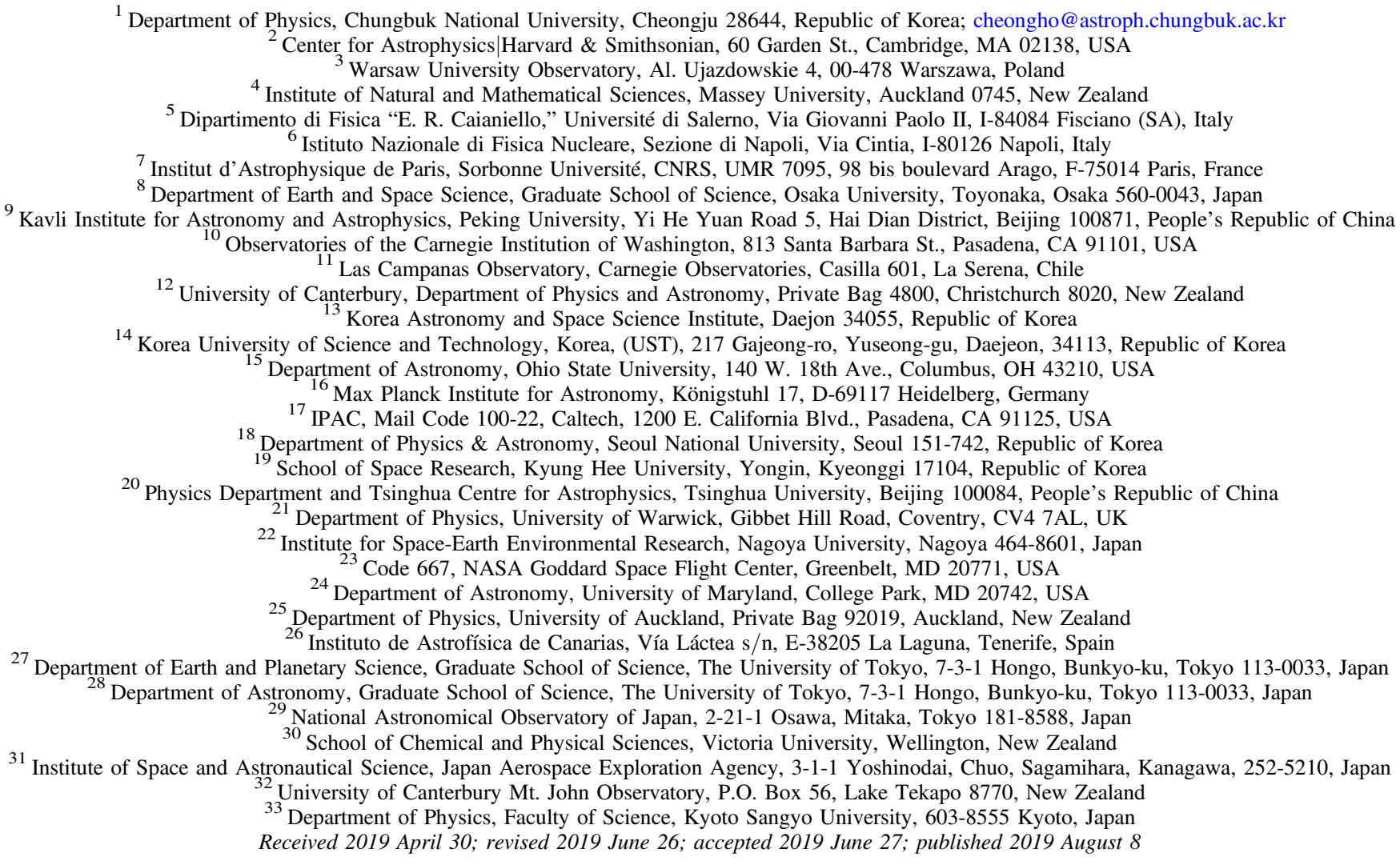

Abstract

We report the discovery of the microlensing planet OGLE-2018-BLG-0740Lb. The planet is detected with a very strong signal of $\Delta \chi^{2} \sim 4630$, but the interpretation of the signal suffers from two types of degeneracies. One type is caused by the previously known close/wide degeneracy, and the other is caused by an ambiguity between two

\footnotetext{
${ }_{35}^{34}$ KMTNet Collaboration.

35 OGLE Collaboration.

36 MOA Collaboration.
} 
solutions, in which one solution requires the incorporation of finite-source effects, while the other solution is consistent with a point-source interpretation. Although difficult to be firmly resolved based on only the photometric data, the degeneracy is resolved in strong favor of the point-source solution with the additional external information obtained from astrometric and spectroscopic observations. The small astrometric offset between the source and baseline object supports that the blend is the lens and this interpretation is further secured by the consistency of the spectroscopic distance estimate of the blend with the lensing parameters of the point-source solution. The estimated mass of the host is $1.0 \pm 0.1 M_{\odot}$ and the mass of the planet is $4.5 \pm 0.6 M_{\mathrm{J}}$ (close solution) or $4.8 \pm 0.6 M_{\mathrm{J}}$ (wide solution) and the lens is located at a distance of $3.2 \pm 0.5 \mathrm{kpc}$. The bright nature of the lens, with $I \sim 17.1(V \sim 18.2)$, combined with its dominance of the observed flux suggest that radialvelocity (RV) follow-up observations of the lens can be done using high-resolution spectrometers mounted on large telescopes, e.g., Very Large Telescope/ESPRESSO, and this can potentially not only measure the period and eccentricity of the planet but also probe for close-in planets. We estimate that the expected RV amplitude would be $\sim 60 \sin i \mathrm{~m} \mathrm{~s}^{-1}$.

Key words: gravitational lensing: micro - planetary systems

\section{Introduction}

Microlensing provides a tool to detect exoplanets because a planetary companion to a lens can manifest its presence through the perturbation to the lensing light curve produced by the host of the planet (Mao \& Paczyński 1991; Gould \& Loeb 1992). The characteristics of the planetary signal varies depending on the lens-system configurations, and the analysis of the signal enables one to determine the planet-host mass ratio, $q$, and the projected planet-host separation in units of the angular Einstein radius $\theta_{\mathrm{E}}, s$. However, determining these planet parameters is often hampered by various types of degeneracy, which lead to multiple interpretations of the observed signal. Finding the types of degeneracies and understanding their origins are important to identify similar degeneracies in subsequent analyses and thus to correctly interpret the observed signal.

The types and origins of degeneracies for some specific cases of planetary signals are known. The "close/wide degeneracy" is the most well-known type, which causes difficulty in distinguishing the perturbations produced by central caustics induced by planetary companions with separations $s$ and $s^{-1}$. This degeneracy is intrinsic in the sense that it is rooted in the symmetry of the lens equations between the lenses with $s$ and $s^{-1}$ (Griest \& Safazadeh 1998; Dominik 1999; An 2005). The "binary-source/planet degeneracy" is an accidental degeneracy, which causes difficulty in distinguishing a short-term planetary anomaly from the anomaly produced by a subset of binary-source events with a small flux ratio between the binarysource stars and the close approach of the faint source companion to the lens (Gaudi 1998). It was recently found that this degeneracy not only applies to a short-term anomaly but also can extend to various cases of planetary lens system configurations (Jung et al. 2017; Dominik et al. 2019; Shin et al. 2019).

With the increasing number of planetary microlensing events, various types of degeneracies have been newly identified. Many of these degeneracies are caused by the ambiguity in determining the exact source trajectory with respect to the caustic. Such an ambiguity was first predicted by Gaudi \& Gould (1997), who pointed out that the magnification pattern on the near and far sides of the major-image caustic, which represented the planetary caustic produced by a planet with $s>1.0$, were similar, and thus the anomalies produced by the source approaching both sides of the caustic were similar to each other: "major-image degeneracy." Han et al. (2018a) pointed out that for some specific lens-system configurations, a similar degeneracy could occur for planetary anomalies produced by the minor-image caustic, which was produced by a planet with $s<1.0$. Skowron et al. (2018) found that a major-image degeneracy could also occur in the case of anomalies resulting from the source star's caustic crossings. This "caustic-chiral degeneracy," which occur when there is a gap in data, results in similar values $s$ but substantially different $q$, while the degeneracy between two non-caustic-crossing degenerate solutions considered by Gaudi \& Gould (1997) results in a similar value of $q$. Hwang et al. (2018) reported a new type of discrete degeneracy between the solution in which the major-image caustic was fully enveloped and the solution in which only one side of the caustic was enveloped. The two solutions subject to this so-called "Hollywood degeneracy" (Gould 1997) result in different mass ratios because the source passes through the caustic in different places relative to its center.

In this paper, we present the analysis of the microlensing event OGLE-2018-BLG-0740, which exhibits a strong shortterm anomaly produced by a planetary companion. We find that the interpretation of the planetary signal suffers from a new type of discrete degeneracy caused by the incomplete coverage of the planetary anomaly. In Section 2, we mention the data acquisition and processing. In Section 3, we describe the procedure of the data analysis and depict the degeneracy found from the analysis. We characterize the source star in Section 4 and present the physical parameters of the planetary system estimated from Bayesian analysis in Section 5. In Section 6, we present the external information that enables us to resolve the degeneracy. In Section 7, we discuss the possibility of further characterizing the planetary system, including measuring the planet's period and eccentricity as well as probing for additional planets, using radial velocity (RV) measurements. In Section 8, we summarize the results and conclude.

\section{Observation and Data}

The source star of the microlensing event OGLE-2018-BLG0740 is located toward the Galactic bulge field with equatorial coordinates (R.A., decl.) $)_{\mathrm{J} 2000}=(18: 08: 42.47,-29: 50: 08.9)$, which correspond to the Galactic coordinates $(l, b)=(1.74$, -4.80 ). The apparent baseline brightness of the star before lensing magnification was $I_{\text {base }} \sim 16.87$. We note that the source is heavily blended as we will show in Section 4 and only $\sim 2 \%$ of the measured flux comes from the source star.

The lensing event was first found by the Optical Gravitational Lensing Experiment (OGLE: Udalski et al. 2015) on 
2018 May $8\left(\mathrm{HJD}^{\prime} \equiv \mathrm{HJD}-2,450,000 \sim 8246\right)$ when the source had apparently brightened by $\sim 0.14$ mag from the baseline, and the discovery of the event was notified to the microlensing community. On 2018 May $16\left(\mathrm{HJD}^{\prime} \sim 8254\right)$, the event was also found by the Microlensing Observations in Astrophysics (MOA) group (Bond et al. 2001; Sumi et al. 2003). In the "MOA Transient Alerts" page, the event was listed as MOA-2018-BLG-147. OGLE observations were conducted mostly in $I$ band, with occasional $V$-band observations for the source-color measurement, with $\sim 1$ day cadence using the $1.3 \mathrm{~m}$ telescope located at Las Campanas Observatory in Chile. MOA observations were carried out in a customized broad $R$ band with $\sim 1 \mathrm{hr}$ cadence using the $1.8 \mathrm{~m}$ telescope located at the Mt. John University Observatory in New Zealand.

The event was independently discovered by the Korea Microlensing Telescope Network (KMTNet: Kim et al. 2016) survey in its annual post-season analysis (Kim et al. 2018) and was designated as KMT-2018-BLG-1822. KMTNet observations were conducted using three identical $1.6 \mathrm{~m}$ telescopes that are located at the Siding Spring Observatory, Australia (KMTA), Cerro Tololo Interamerican Observatory, Chile (KMTC), and the South African Astronomical Observatory, South Africa (KMTS). During the period near the anomaly, KMTNet observed this field with cadence of $2.5 \mathrm{hr}$ from KMTC and $3.3 \mathrm{hr}$ from KMTA and KMTS. KMTNet observations were conducted both in $I$ and $V$ bands, and onetenth of KMT I-band observations are complemented by $V$ band images.

In addition to the usual photometric observations, additional spectroscopic follow-up observations were conducted using the Inamori Magellan Areal Camera and Spectrograph (IMACS, Dressler et al. 2011) mounted on the $6.5 \mathrm{~m}$ Magellan-Baade telescope at Las Campanas Observatory in Chile. As will be discussed in Section 4, the spectroscopic observations were conducted to specify the stellar type of the blend object.

The event was analyzed in real time with its progress. On 2018 May $14\left(\mathrm{HJD}^{\prime} \sim 8252\right)$, V. Bozza noticed a single point anomaly at $\mathrm{HJD}^{\prime}=8250.89$ in the OGLE data, which was confirmed to be real by the OGLE group. The anomaly was additionally confirmed in real-time by the MOA ( 3 points) and later by KMTNet data sets ( 3 KMTC and 2 KMTA points). Since the anomaly was confirmed, a series of models describing the anomaly were circulated by V. Bozza, A. Cassan, and Y. Hirao. Although there existed slight variation in detailed lensing parameters, all models agreed that the anomaly was produced by a planetary companion.

Photometric data sets used in the analysis are processed using the photometry codes of the individual survey groups: Udalski (2003), Bond et al. (2001), and Albrow et al. (2009) for the OGLE, MOA, and KMTNet surveys, respectively. All of these codes are based on the difference imaging technique developed by Alard \& Lupton (1998). We normalize the error bars of the individual data sets following the procedure described in Yee et al. (2012). In order to measure the source color, we additionally conduct photometry using the pyDIA photometry (Albrow 2017) for a subset of the KMTNet data (KMTC $I$ - and $V$-band data sets).

In Figure 1, we present the light curve of the lensing event. The curve superposed on the observed data points represents the model based on the single-lens (1L) and single-source (1S) modeling excluding the anomaly part of the data. The inset

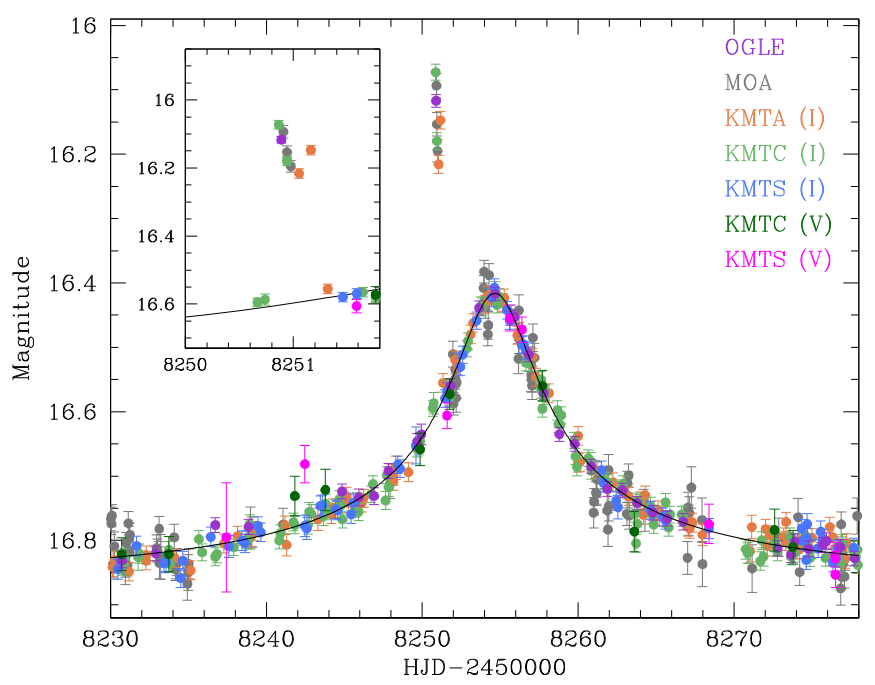

Figure 1. Light curve of the microlensing event OGLE-2018-BLG-0740. The solid curve superposed on the data points represents the single-lens and singlesource (1L1S) model obtained by fitting the data excluding those around the anomaly at $\mathrm{HJD}^{\prime} \sim 8251$. The inset shows the enlarged view of the anomaly.

shows the enlarged view of the anomaly, which occurred at $t_{\text {anom }} \sim 8251$. The duration of the anomaly, which lasted less than a day, is short. Apart from the anomaly, one finds that the event is well described by a $1 \mathrm{~L} 1 \mathrm{~S}$ model.

\section{Interpretation of the Anomaly}

For the interpretation of the anomaly, we conduct modeling of the light curve. The observed short-term anomaly is a characteristic feature produced by a planetary companion to the lens, and thus we first conduct modeling under the assumption that the lens is composed of two masses: $2 \mathrm{~L} 1 \mathrm{~S}$ model. Because it is known that such an anomaly could in principle also be produced by a companion to a source, we also conduct modeling under the binary-source assumption: 1L2S model.

\subsection{L1S Modeling}

A short-term planetary microlensing signal is produced by the passage over or approach of the source close to the caustic induced by the planet. The planet-induced caustics are classified into two types: "central" and "planetary" caustics. The central caustic is located close to the primary lens, while the planetary caustic is located away from the primary with a separation $\sim s-s^{-1}$. For the properties of the planet-induced caustic, see Chung et al. (2005) and Han (2006) for the central and planetary caustics, respectively.

Under the planetary interpretation of the anomaly, one can heuristically characterize the planet. The values of $\left(t_{0}, u_{0}\right.$, $\left.t_{\mathrm{E}}\right) \sim(8254.6,0.035,71$ days $)$ obtained from the $1 \mathrm{~L} 1 \mathrm{~S}$ modeling for the data excluding the anomaly, together with $t_{\text {anom }} \sim 8251.0$, where the times $t_{0}$ and $t_{\text {anom }}$ are expressed in $\mathrm{HJD}^{\prime} \equiv \mathrm{HJD}-2,450,000$, indicate that the caustic is located relatively close to the primary, and thus the perturbation is likely to be produced by the central caustic rather than the planetary caustic. One can estimate the source trajectory angle $\alpha$ (with respect to the binary axis) from the relation

$$
\alpha=\tan ^{-1}\left(\frac{u_{0} t_{\mathrm{E}}}{t_{\text {anom }}-t_{0}}\right) \sim 2.5 \text { radian },
$$


Table 1

Lensing Parameters of Planetary Solutions

\begin{tabular}{lcccc}
\hline \hline Parameter & Close+Finite & Close + Point & Wide+Finite & Wide + Point \\
\hline$\chi^{2}$ & 1265.4 & 1245.5 & 1267.5 & 1244.4 \\
$t_{0}\left(\mathrm{HJD}^{\prime}\right)$ & $8254.347 \pm 0.031$ & $8254.242 \pm 0.032$ & $8254.351 \pm 0.029$ & $8254.223 \pm 0.033$ \\
$u_{0}$ & $0.038 \pm 0.003$ & $0.036 \pm 0.003$ & $0.038 \pm 0.003$ & $0.033 \pm 0.003$ \\
$t_{\mathrm{E}}$ (days) & $62.63 \pm 4.37$ & $64.58 \pm 4.24$ & $1.18 \pm 0.01$ & $70.12 \pm 5.16$ \\
$s$ & $0.91 \pm 0.01$ & $0.86 \pm 0.01$ & $2.13 \pm 0.17$ & $1.26 \pm 0.01$ \\
$q\left(10^{-3}\right)$ & $2.30 \pm 0.18$ & $4.28 \pm 0.41$ & $2.508 \pm 0.008$ & $4.54 \pm 0.51$ \\
$\alpha(\mathrm{rad})$ & $2.520 \pm 0.009$ & $2.517 \pm 0.009$ & $0.85 \pm 0.08$ & $<12 \pm 0.010$ \\
$\rho\left(10^{-3}\right)$ & $0.99 \pm 0.10$ & $<0.4$ & $<0.5$ \\
\hline
\end{tabular}

Note. $\mathrm{HJD}^{\prime} \equiv \mathrm{HJD}-2,450,000$.

which is very similar to the value obtained from detailed modeling described below.

We conduct systematic analysis of the observed data to search for the exact lensing parameters. In the first step of this analysis, we conduct grid searches for the binary-lens parameters $(s, q)$, while the other lensing parameters $\left(t_{0}, u_{0}\right.$, $\left.t_{\mathrm{E}}, \alpha\right)$ are searched for using a downhill method of the Markov Chain Monte Carlo (MCMC) algorithm (Goodman \& Weare 2010). For the parameters $\left(t_{0}, u_{0}, t_{\mathrm{E}}\right)$, we use the values obtained from the 1L1S modeling as initial parameters. For the source trajectory angle, we seed 21 different initial values around a unit circle. The sudden change of the source brightness $(\Delta I \sim 0.4)$ before and after the perturbation suggests that the perturbation was produced by the caustic crossings of the source. We, therefore, include an additional parameter of $\rho$ (normalized source radius), which represents the ratio of the angular source radius $\theta_{*}$ to $\theta_{\mathrm{E}}$, i.e., $\rho=\theta_{*} / \theta_{\mathrm{E}}$, to account for finite-source effects that affect the light curve during caustic crossings. From this first step analysis, we identify local minima in the $\Delta \chi^{2}$ plot on the plane of the grid parameters, i.e., $s-q$ plane. In the second step, we refine the individual local solutions by allowing all parameters, both the grid parameters $(s, q)$ and the MCMC parameters $\left(t_{0}, u_{0}, t_{\mathrm{E}}, \alpha, \rho\right)$, to vary.

From the 2L1S modeling, we identify four discrete degenerate solutions. In Table 1, we list the lensing parameters of these solutions, together with their $\chi^{2}$ values. The mass ratios for all of the solutions are $q<10^{-2}$, indicating that the lens is a planetary system. The planetary solutions greatly improve the fit by $\Delta \chi^{2} \sim 4630$ with respect to the $1 \mathrm{~L} 1 \mathrm{~S}$ solution. Despite the very strong planetary signal, the $\chi^{2}$ differences between the degenerate solutions are merely $\Delta \chi^{2} \lesssim 23$, and thus the degeneracy is substantial. In Figure 2, we present the model light curves of the individual solutions in the region of the anomaly. In Figure 3, we also present the lens-system configurations, which show the source trajectories with respect to the caustic, of the individual solutions. As expected from the location of the anomaly lying close to the peak of the light curve and the strong deviation from the 1L1S model, the anomaly is produced by the crossing of the source over the central caustic induced by the planetary companion for all cases of the degenerate solutions.

We inspect the origin of the degeneracy and find that there exist two types of degeneracies. The first degeneracy is caused by the uncertain planet-host separation. This is shown in the upper panel of Figure 4, in which we present the $\Delta \chi^{2}$ distribution of MCMC points on the $s-q$ plane. From the locations of the local solutions, it is found that two solutions have similar mass ratios of $q \sim 5 \times 10^{-3}$ and the other two

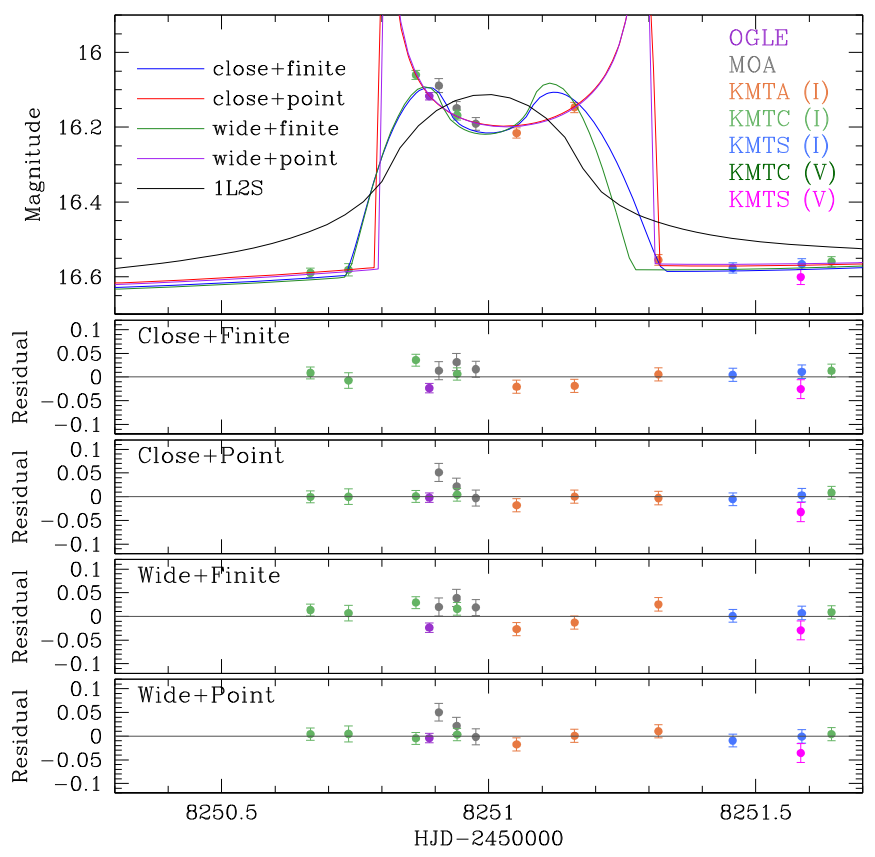

Figure 2. Comparison of model light curves in the region of the anomaly. In the top panel, the curves marked in blue, red, green, and purple colors are models of the "close+finite," "close+point," "wide+finite," and "wide +point," respectively, which are the four degenerate solutions found from 2L1S modeling. The lower four panels show the residuals from the individual solutions. The black curve is the solution obtained from the 1L2S modeling.

solutions have mass ratios $q \sim 2 \times 10^{-3}$. For the pair of solutions with similar mass ratios, it is found that one solution has a separation $s<1.0$, while the other solution has a separation $s>1.0$. This indicates that the degeneracy between the pair of solutions with $s<1.0$ and $s>1.0$ is caused by the well-known "close/wide degeneracy." For planetary lens systems with very low mass ratios and projected separations substantially greater or smaller than unity, the planetary and central caustics are well separated. In such cases, the projected separations of the two degenerate solutions subject to the close/wide degeneracy are in the relation of $s \leftrightarrow s^{-1}$. In the case of OGLE-2018-BLG-0740, the projected separations of the pairs of the degenerate solutions slightly deviate from this relation because the separations are close to unity: $s \sim 0.9$ for the close solutions and $s \sim 1.2$ for the wide solutions.

We find that the other degeneracy arises due to the ambiguity in the normalized source radius, $\rho$, caused by the incomplete coverage of the anomaly. To show this, we mark the positions of the local solutions on the $\rho-q$ parameter plane presented in the lower panel of Figure 4. It is found that there exist two 


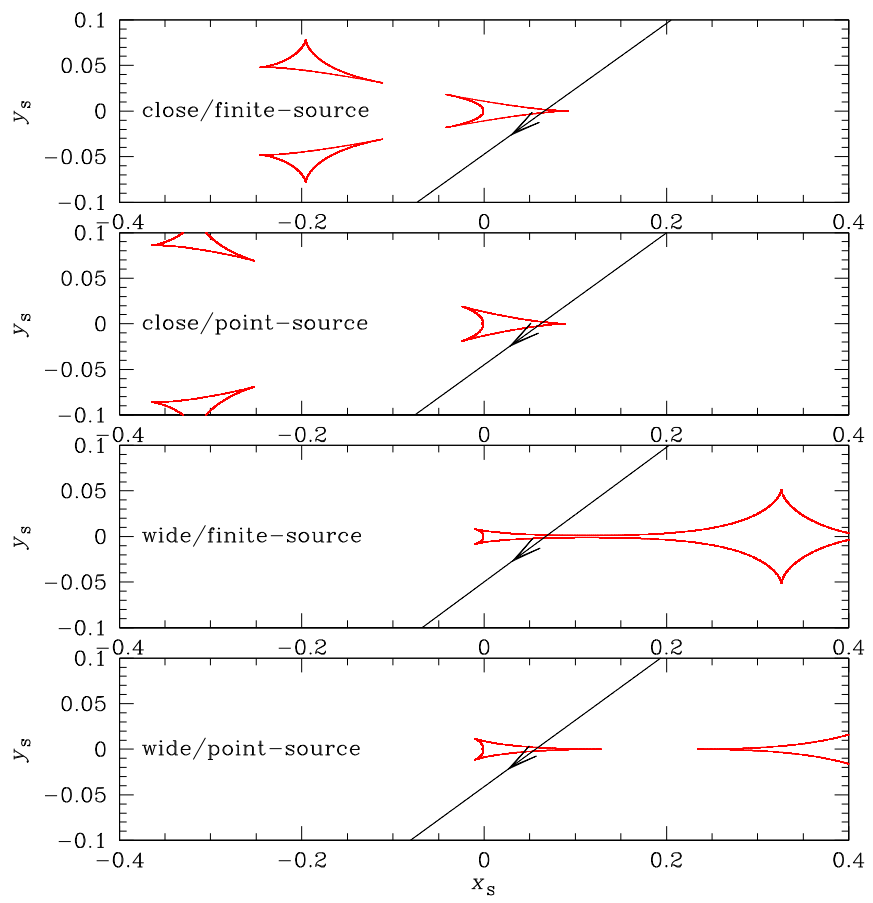

Figure 3. Lens-system configurations of the four degenerate solutions found from 2L1S modeling. In each panel, the line with an arrow represents the source trajectory and the closed figures composed of concave curves are the caustics. The lensing parameters corresponding to the individual solutions are presented in Table 1.
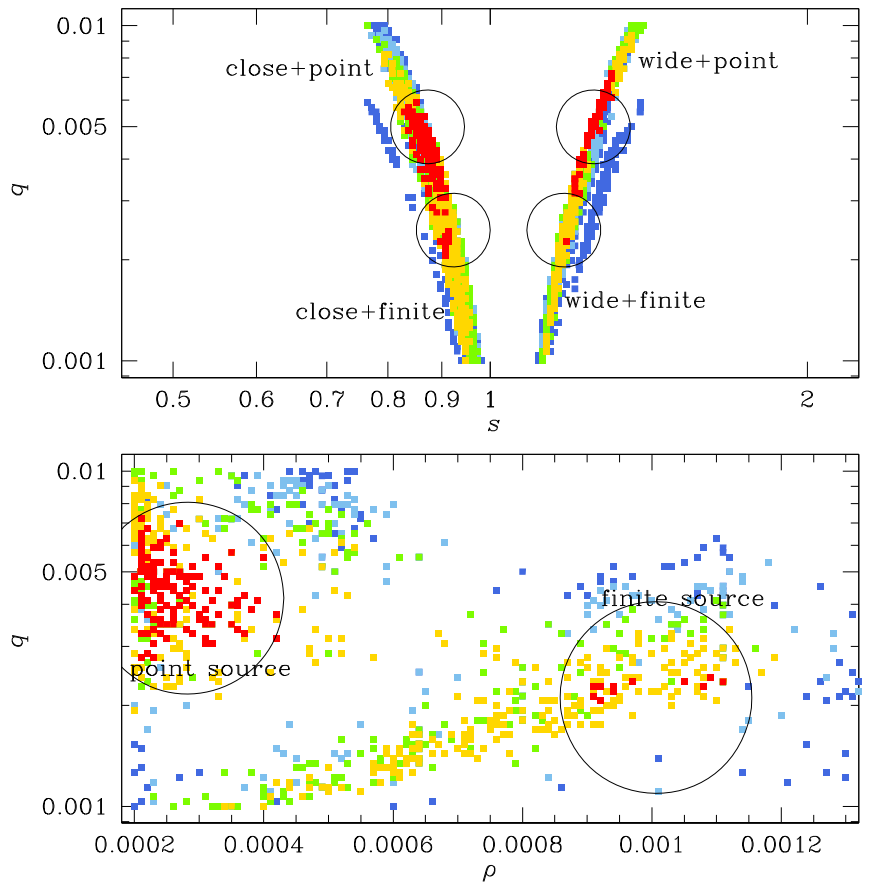

Figure 4. $\Delta \chi^{2}$ distributions of points in the MCMC chain on the $s-q$ (upper panel) and $\rho-q$ (lower panel) planes. Red, yellow, green, cyan, and blue colors are used to indicate points with $1 n \sigma, 2 n \sigma, 3 n \sigma, 4 n \sigma$, and $5 n \sigma$, respectively, and $n=5$.

locals, in which the one with a smaller mass ratio, i.e., $q \sim 2 \times 10^{-3}$, has a normalized source radius of $\rho \sim 1.0 \times 10^{-3}$, while the normalized source radius of the other local with a larger mass ratio, i.e., $q \sim 5 \times 10^{-3}$, is consistent with zero, i.e., point source. We refer to this
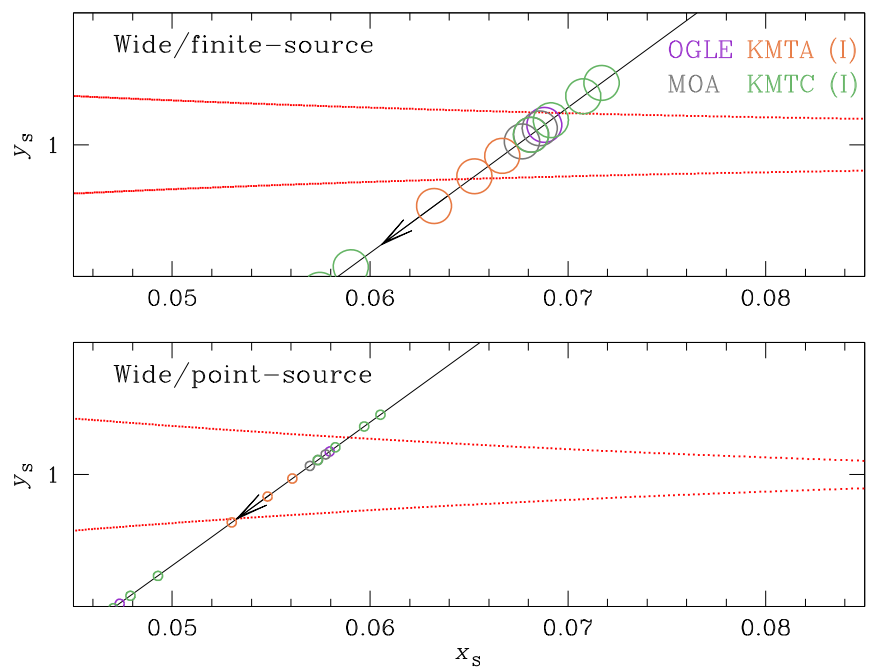

Figure 5. Source positions at around the time of the anomaly. The upper and lower panels correspond to the "close/finite-source" and "close/point-source" solutions, respectively. In each panel, the line with an arrow is the source trajectory and the red curves represent the caustic. The small circles on the source trajectory represent the source size. Although the source is consistent with a point for the point-source solution, we show it with normalized radius $\rho=2.3 \times 10^{-4}$ for reasons discussed in Section 6 .

degeneracy as the "finite/point-source degeneracy." The fact that the solutions with different $\rho$ values have different values of $s$ and $q$ indicates that the finite/point-source degeneracy causes ambiguity in the determinations of both $s$ and $q$, while the close/wide degeneracy causes ambiguity in the determination of only $s$.

We designate the individual local solutions as "close +finite," "close+point," "wide+finite," and "wide+point." Here the terms "close" and "wide" indicate $s<1$ and $s>1$, respectively. The term "finite" is used to represent that the light curve is subject to finite-source effects, while the term "point" is used to represent that the light curve is consistent with that of a point-source event. According to the point-source solutions, the source positions corresponding to the anomalous data points are well within the caustic and the data points during the anomaly are placed in the "U"-shape trough region between the caustic-crossing spikes of the light curve. According to the finite-source solutions, on the other hand, most data points correspond to the source positions during which the source was crossing the caustic. See the data points around the anomaly region of the light curve presented in Figure 2 and the corresponding source positions presented in Figure 5.

In order to see the severity of the "finite/point-source" degeneracy, in Figure 6, we present the cumulative distribution of $\chi^{2}$ difference between the "finite-source" and "point-source" solutions in the region of the anomaly. We note that the plot is for the pair of the wide solutions with $s>1.0$, but for the pair of the close solutions with $s<1.0$, the plot is very similar to the presented $\Delta \chi^{2}$ distribution. From the $\Delta \chi^{2}$ plot, it is found that the point-source solution is favored over the finite-source solution by $\Delta \chi^{2} \sim 18$ during the anomaly. However, considering that this level of $\Delta \chi^{2}$ might be caused by systematics in microlensing data together with the fact that the $\chi^{2}$ difference originates mainly from a few points, it is difficult to completely rule out the finite-source solution just based on the observed photometric data.

We inspect the higher-order effects in the observed light curve caused by the orbital motion of the observer, microlens- 


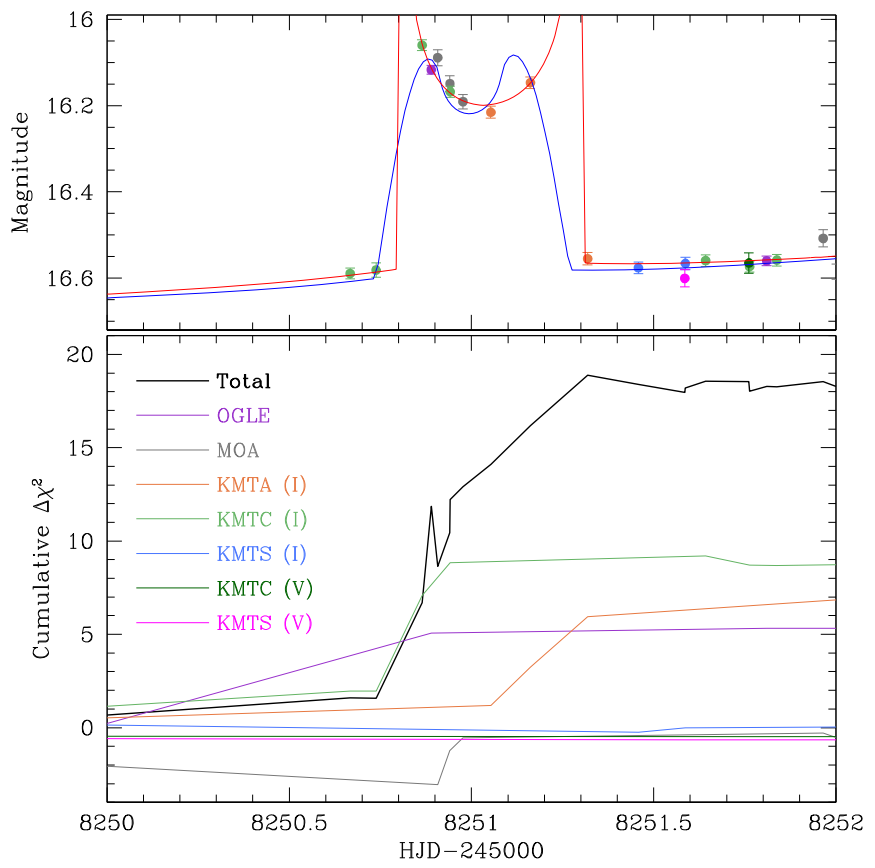

Figure 6. Cumulative distribution of $\Delta \chi^{2}$ between the "finite" and "point" solutions (with $s<1.0$ ) in the region of the anomaly. The black curve is the distribution for all data sets, and the distributions for the individual data sets are marked in different colors that match those of the legends. The light curve in the upper panel is presented to show the region of $\chi^{2}$ difference.

parallax effects (Gould 1992), and the orbital motion of the lens, lens-orbital effects (Dominik 1998). For this inspection, we conduct a series of modeling separately and simultaneously considering these effects. We find that these modeling runs result in little improvement of the fit, with $\Delta \chi^{2} \sim 2$ when both the higher-order effects are simultaneously considered. The difficulty of measuring the higher-order effects arises because the source is a faint star with $I \sim 21.5$ (see Section 4), and thus the photometric quality is not high enough to detect subtle deviations induced by the higher-order effects, despite the relatively long timescale, $t_{\mathrm{E}} \gtrsim 60$ days, of the event.

\subsection{IL2S Modeling}

Because it is known that a short-term anomaly can also be produced by a binary companion to the source, we additionally conduct a 1L2S modeling. Besides the 1L1S lensing parameters of $\left(t_{0}, u_{0}, t_{\mathrm{E}}\right)$, this modeling requires the inclusion of additional parameters of $\left(t_{0,2}, u_{0,2}, q_{F}\right)$, where $t_{0,2}$ is the time of the closest lens approach to the source companion, $u_{0,2}$ is the lens-companion separation at $t_{0,2}$, and $q_{F}$ represents the flux ratio between the two source stars. For the initial value of $t_{0,2}$, we use the time of the anomaly, $t_{\text {anom. }}$. We set the initial values of $u_{0,2}$ and $q_{F}$ considering that the source has a small flux ratio, $q_{F} \ll 1$, and the lens approaches very close to the source companion, $u_{0,2} \rightarrow 0$, for $1 \mathrm{~L} 2 \mathrm{~S}$ events producing short-term anomalies.

In the upper panel of Figure 2, we present the model light curve of the best-fit $1 \mathrm{~L} 2 \mathrm{~S}$ solution. It is found that the model provides a poorer fit than the $2 \mathrm{~L} 1 \mathrm{~S}$ solutions. The $\chi^{2}$ difference between the 1L2S and 2L1S solutions is $\Delta \chi^{2} \sim 606$. We, therefore, reject this interpretation of the anomaly and conclude that the origin of the anomaly is the planetary companion to the lens.

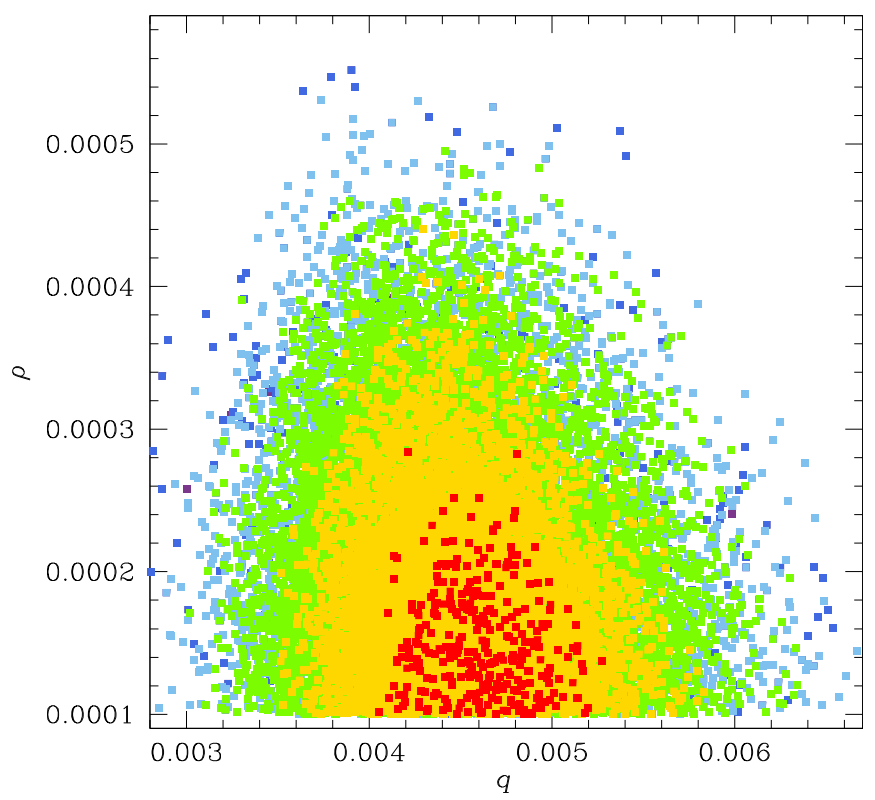

Figure 7. $\Delta \chi^{2}$ distribution of points in the MCMC chain on the $q-\rho$ plane for the "wide+point" solution. Color coding is set to represent points within $1 \sigma$ (red), $2 \sigma$ (yellow), $3 \sigma$ (green), $4 \sigma$ (cyan), and $5 \sigma$ (blue) from the best-fit value.

\section{Source and Blend}

We characterize the source star based on its dereddened color $(V-I)_{0}$ and brightness $I_{0}$. For the determinations of $(V-I)_{0}$ and $I_{0}$, we use the method of Yoo et al. (2004), which utilizes the centroid of the red giant clump (RGC) in the colormagnitude diagram (CMD) as a reference to calibrate the color and brightness of the source star. Defining the source star is important for the determination of the angular Einstein radius because $\theta_{\mathrm{E}}$ is related to the angular radius of the source star, $\theta_{*}$, by the relation

$$
\theta_{\mathrm{E}}=\frac{\theta_{*}}{\rho},
$$

where $\theta_{*}$ is estimated from the source type and the normalized source radius $\rho$ is measured by analyzing the caustic-crossing parts of the light curve.

We note that the angular Einstein radius can be measured from the relation in Equation (2) for the "finite-source" solutions because the $\rho$ value is measured for these solutions, but the value of $\rho$ is not measured for the "point-source" solutions and thus $\theta_{\mathrm{E}}$ cannot be measured. For the "pointsource" solutions, however, one can set the lower limit of the angular Einstein radius by

$$
\theta_{\mathrm{E}, \min }=\frac{\theta_{*}}{\rho_{\max }},
$$

where $\rho_{\max }$ represents the upper limit of the normalized source radius. In Figure 7 , we present the $\Delta \chi^{2}$ distribution of the points in the MCMC chain on the $q-\rho$ plane for the "wide +point" solution. It shows that the upper limit is $\rho_{\max } \sim 0.5 \times 10^{-3}$ as measured at the $3 \sigma$ level. The "close + point" solution yields a similar value of $\rho_{\text {max }}$.

In Figure 8, we mark the location of the source (empty circle with error bars) in the CMD of stars around the source. Also marked are the locations of the RGC centroid (red dot) and blend (green dot). We determine the $I$ - and $V$-band magnitudes 


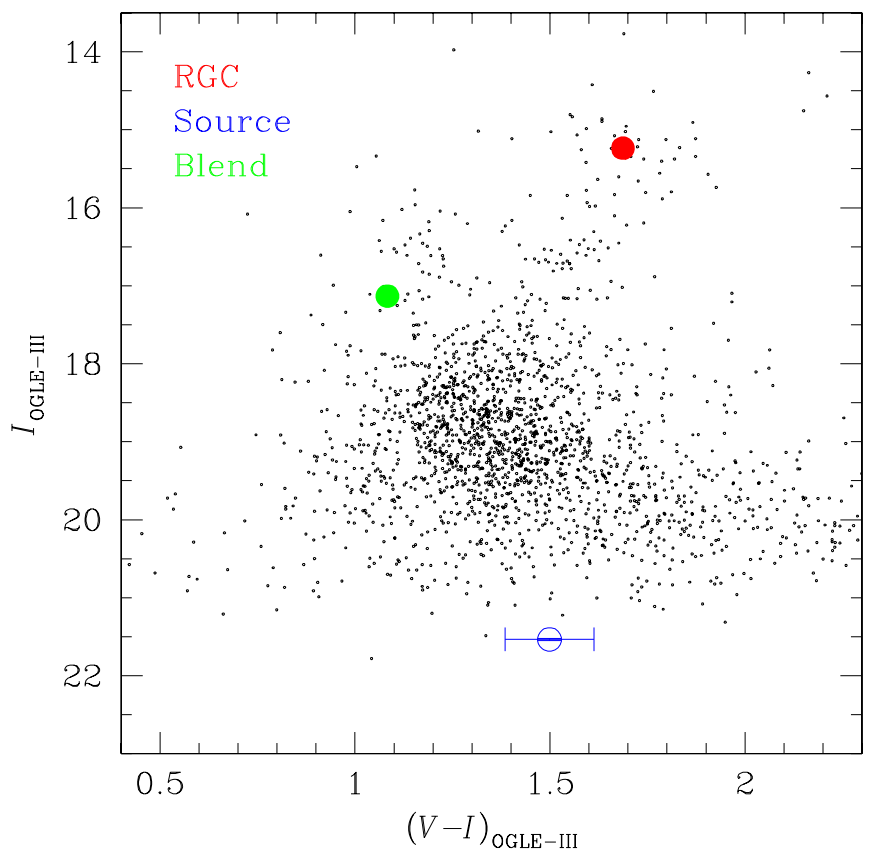

Figure 8. Locations of the source and blend in the color-magnitude diagram with respect to the centroid of red giant clump (RGC). The color and magnitude are estimated based on the KMTC data set, but they are calibrated to OGLE-III photometry.

of the source using the regression method for the KMTC photometry data processed with the pyDIA photometry. We note that the KMTC data are not calibrated, and thus the color and magnitude are scaled to those of OGLE-III CMD (Szymański et al. 2011) in order to estimate calibrated color and brightness. The apparent color and brightness of the source are $(V-I, I)=(1.49 \pm 0.11,21.54 \pm 0.01)$. From the offset of $\Delta(V-I, I)=(-0.20,6.30)$ with respect to the RGC centroid at $(V-I, I)_{\mathrm{RGC}}=(1.69,15.24)$, together with the known dereddened color and brightness of the RGC centroid of $(V-I$, $I)_{\mathrm{RGC}, 0}=(1.06,14.35)$ (Bensby et al. 2011; Nataf et al. 2013), we estimate that the dereddened color and brightness of the source stars are $(V-I, I)_{0}=(0.87 \pm 0.11,21.53 \pm 0.01)$. This indicates that the source is an early K-type main-sequence star. Once the dereddened $V-I$ color is measured, we then convert $V-I$ color into $V-K$ color using the color-color relation of Bessell \& Brett (1988), and then estimate the angular radius of the source using the $(V-K) / \theta_{*}$ relation of Kervella et al. (2004). The estimated angular source radius from this procedure is

$$
\theta_{*}=0.28 \pm 0.04 \mu \mathrm{as} \text {. }
$$

With the measured angular source radius, the angular Einstein radius, $\theta_{\mathrm{E}}$, and the relative lens-source proper motion, $\mu=\theta_{\mathrm{E}} / t_{\mathrm{E}}$, are determined. These values are

$$
\begin{aligned}
\theta_{\mathrm{E}, \mathrm{FS}} & =0.28 \pm 0.04 \text { mas }, \\
\mu_{\mathrm{FS}} & =1.65 \pm 0.23 \text { mas } \mathrm{yr}^{-1}
\end{aligned}
$$

for the "finite-source" solutions. We note that the estimated values of $\theta_{\mathrm{E}}$ and $\mu$ for the finite-source solutions are substantially lower than $\left\langle\theta_{\mathrm{E}}\right\rangle \sim 0.5$ mas and

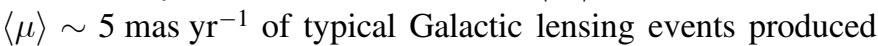
by low-mass stars, $\sim 0.3 M_{\odot}$, located halfway between the source and observer, $D_{\mathrm{L}} \sim 4 \mathrm{kpc}$, in the disk. If the finitesource solution were correct, then the lens would very likely lie
Table 2

Lens Parameters from Bayesian Analysis

\begin{tabular}{lcc}
\hline \hline Parameter & Point-source & Finite-source \\
\hline$\theta_{\mathrm{E}}(\mathrm{mas})$ & $>0.56$ & $0.28 \pm 0.04$ \\
$\mu\left(\mathrm{mas}^{-1}\right)$ & $>2.9$ & $1.65 \pm 0.23$ \\
$M_{\mathrm{host}}\left(M_{\odot}\right)$ & $0.83_{-0.33}^{+0.47}$ & $0.47_{-0.24}^{+0.31}$ \\
$M_{\mathrm{p}}\left(M_{\mathrm{J}}\right)$ & $3.9_{-1.6}^{+2.2}$ & $1.1_{-0.6}^{+0.7}$ \\
$D_{\mathrm{L}}(\mathrm{kpc})$ & $4.2_{-1.6}^{+1.6}$ & $7.2_{-1.0}^{+0.9}$ \\
$a_{\perp}(\mathrm{au})$ & $3.3_{-1.3}^{+1.3}$ (close) & $1.5_{-0.2}^{+0.2}$ (close) \\
& $4.6_{-1.8}^{+1.8}$ (wide) & $2.0_{-0.3}^{+0.2}$ (wide)
\end{tabular}

Note. The two values of the projected planet-host separation $a_{\perp}$ correspond to the close and wide solutions.

in the bulge both because of the small Einstein radius and low proper motion.

For the "point-source" solutions, for which only the upper limit of $\rho$ is determined, the corresponding lower limits are

$$
\theta_{\mathrm{E}, \mathrm{PS}}>0.56 \text { mas, } \quad \mu_{\mathrm{PS}}>2.9 \operatorname{mas}_{\mathrm{yr}^{-1}} \text {. }
$$

These values are reasonably consistent with those of typical disk lens events. See Section 5 for the detailed discussion of the probable lens locations for the individual solutions based on microlensing data alone, and see Section 6 for the final determination of the lens distance using external data. In Table 2, we list the estimated values of $\theta_{\mathrm{E}}$ and $\mu$ for the finitesource and point-source solutions.

The observed flux is dominated by blended light and thus we also characterize the blend. Another reason for identifying the blend is to check the possibility that the blend is the lens such as in the case of OGLE-2017-BLG-0039 (Han et al. 2018b). The apparent color and brightness of the blend for OGLE2018-BLG-0740 are $(V-I, I)_{\mathrm{b}}=(1.08,17.13)$. Considering the color and brightness, the blend is likely to be a mainsequence star located in the disk. The extinction and reddening toward the field are $A_{I} \sim 0.79$ and $E(V-I) \sim 0.66$, respectively (Gonzalez et al. 2012). Assuming that the blend experiences $\sim 1 / 2$ of the total extinction and reddening toward the bulge field, the dereddened color of the blend is estimated as $(V-I)_{0, \mathrm{~b}} \sim(V-I)_{\mathrm{b}}-E(V-I) / 2 \sim 0.75$. This corresponds to the color of a G-type star.

The dominance of the observed flux by the blended light combined with the bright nature of the blend object enable us to characterize the blend based on the spectra obtained from follow-up observations. The spectroscopic follow-up observations were conducted using the IMACS spectrograph of the Magellan-Baade telescope on UT 2019 March 22 08:40 with 5 minutes of exposure. We chose the 300 lines/nm grism with 17.5 degrees of Blaze Angle (i.e., dispersion of $1.341 \AA /$ pixel) of the $f / 2$ channel and the $0 . ! 9$ slit. In Figure 9, we present the spectrum of the baseline object along with the synthetic spectra of stars from the BOSZ spectral library37 with $T_{\text {eff }}=5000 \mathrm{~K}$, $T_{\text {eff }}=6000 \mathrm{~K}$, and $T_{\text {eff }}=7000 \mathrm{~K}$, which correspond to those of early K-, G-, and F-type stars, respectively. By fitting the blue portion of the IMACS spectrum as a function of parameters interpolated over the ELODIE 3.2 library (Moultaka et al. 2004) of stellar spectra using the University of Lyon Spectroscopic analysis Software (Ulyss) code (Koleva et al. 2009; Wu et al. 2011), we find that $T_{\text {eff }}=5912 \pm 49 \mathrm{~K}$, $\log g=4.5 \pm 0.1$, and $[\mathrm{Fe} / \mathrm{H}]=-0.24 \pm 0.05$, indicating that the blend is an early G-type star, very similar to the Sun 


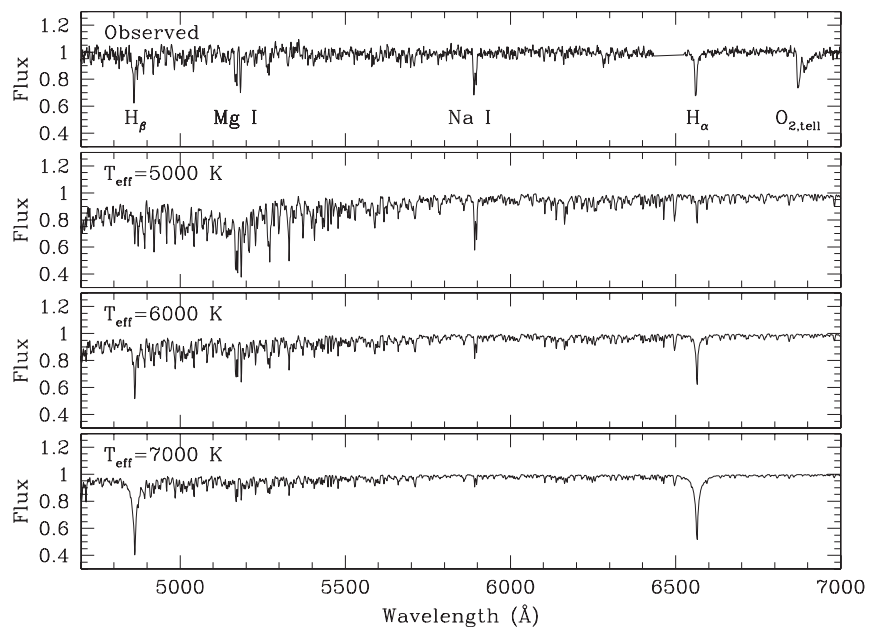

Figure 9. Observed spectrum of the baseline object (top panel). The three lower panels show the synthetic spectra of stars with $T_{\text {eff }}=5000 \mathrm{~K}$, $T_{\text {eff }}=6000 \mathrm{~K}$, and $T_{\text {eff }}=7000 \mathrm{~K}$, which correspond to those of early K-, G-, and F-type stars, respectively. We mark the major spectral lines used to

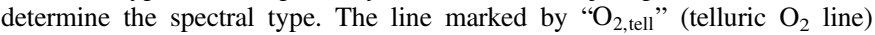
indicates the oxygen molecular line produced by the Earth's atmosphere.

in temperature and gravity, though slightly lower in metallicity. This is consistent with the spectral type estimated based on the photometric data.

\section{Physical Lens Parameters (Bayesian Analysis)}

The mass $M$ and distance $D_{\mathrm{L}}$ to a lens can be uniquely determined when both the angular Einstein radius $\theta_{\mathrm{E}}$ and the microlens parallax $\pi_{\mathrm{E}}$ are simultaneously measured, i.e.,

$$
M=\frac{\theta_{\mathrm{E}}}{\kappa \pi_{\mathrm{E}}} ; \quad D_{\mathrm{L}}=\frac{\mathrm{au}}{\pi_{\mathrm{E}} \theta_{\mathrm{E}}+\pi_{\mathrm{S}}},
$$

where $\kappa=4 G /\left(c^{2} \mathrm{au}\right), \pi_{\mathrm{S}}=\mathrm{au} / D_{\mathrm{S}}$, and $D_{\mathrm{S}}$ denotes the distance to the source. For OGLE-2018-BLG-0740, the angular Einstein radius is measured for the finite-source solutions and the lower limit is constrained for the point-source solutions, but the microlens parallax is not measured for either of these solutions. We, therefore, estimate the physical lens parameters by conducting a Bayesian analysis of the event with the constraints of the measured event timescale and angular Einstein radius.

The Bayesian analysis is carried out by producing a large number of events, $10^{6}$, from a Monte Carlo simulation based on the prior conditions of lens mass composition, i.e., mass function, and the distributions of astronomical objects and their motion, i.e., physical and dynamical distributions, respectively. For the mass function, we adopt the Chabrier (2003) model for stars and the Gould (2000) model for stellar remnants. We adopt the Han \& Gould (2003) model for the physical distribution of matter in the Galaxy and the Han \& Gould (1995) model for the dynamical distribution. A more detailed description of the adopted prior models can be found in Section 5 of Han et al. (2018a). From the probability distributions of the physical parameters for events with timescales and Einstein radii within the ranges of the measured values, we estimate the physical parameters and their uncertainties.

In Figure 10, we present the probability distributions of the host mass and the distance to the lens constructed from the Bayesian analysis. Because there exist two classes of
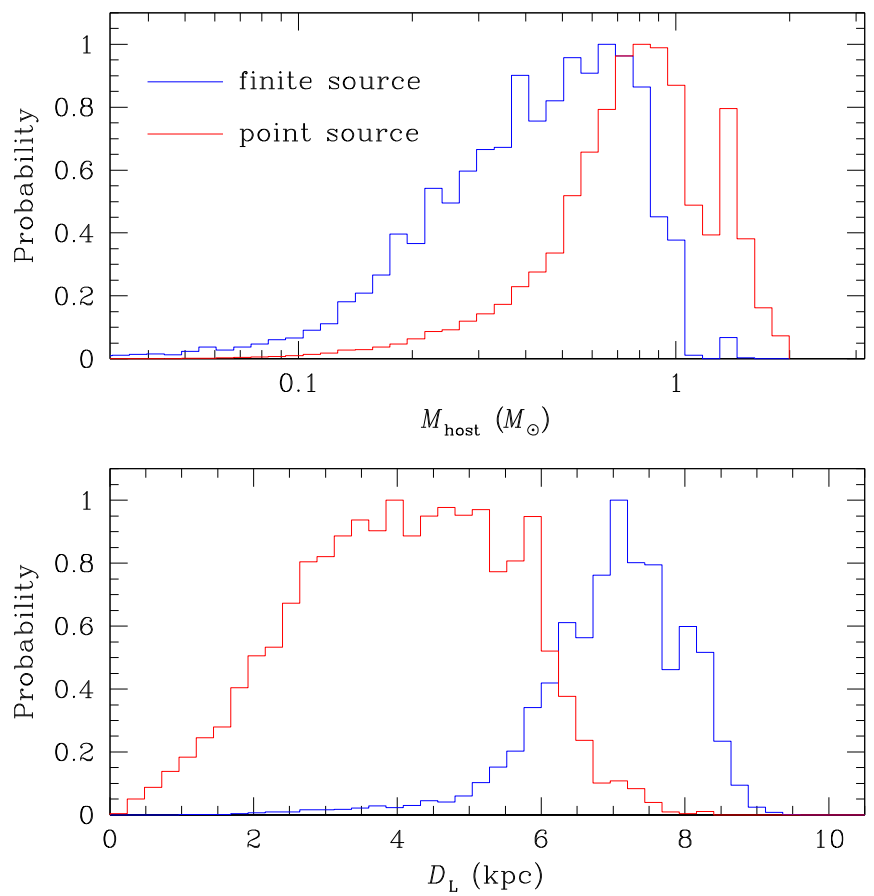

Figure 10. Probability distributions of the lens mass (upper panel) and distance (lower panel). The blue and red curves are the distributions for the finite and point-source solutions, respectively.

degenerate solutions, i.e., finite-source and point-source solutions, we present two probability distributions corresponding to the individual classes of solutions. In Table 2, we present the estimated physical parameters of the lens system, including the host mass, $M_{\text {host }}$, planet mass, $M_{\mathrm{p}}$, distance to the lens, $D_{\mathrm{L}}$, and the physical projected separation between the host and planet, $a_{\perp}$. We note that the presented physical parameters correspond to the median values of the probability distributions and their lower and upper limits are estimated as the $16 \%$ and $84 \%$ of the distributions, respectively. We note that the two values of $a_{\perp}$ correspond to the close and wide solutions.

Note that, for the lens parameters, the physical parameters estimated from the two classes of the degenerate solutions are substantially different. According to the point-source solutions, the masses of the host and planet are

$$
M_{\text {host }}=0.83_{-0.33}^{+0.47} M_{\odot},
$$

and

$$
M_{\mathrm{p}}=3.9_{-1.6}^{+2.2} M_{\mathrm{J}}
$$

respectively, and the distance to the lens is

$$
D_{\mathrm{L}}=4.2_{-1.6}^{+1.6} \mathrm{kpc} \text {. }
$$

In this case, the planetary system consists of a super-Jupiter mass planet and a $\mathrm{G}-\mathrm{K}$ type host star located more likely in the disk. According to the finite-source solutions, on the other hand, the masses of the lens components are

$$
M_{\text {host }}=0.47_{-0.24}^{+0.31} M_{\odot}
$$

and

$$
M_{\mathrm{p}}=1.1_{-0.6}^{+0.7} M_{\mathrm{J}}
$$


respectively. The lens is located at a distance of

$$
D_{\mathrm{L}}=7.2_{-1.0}^{+0.9} \mathrm{kpc} .
$$

Then, the lens is a planetary system composed of a planet with a mass similar to that of Jupiter and an M-dwarf host located in the Galactic bulge. The differences between the physical parameters for the two classes of solutions indicate that the degeneracy reported in this work greatly affects the determinations of the lens parameters.

\section{Resolving the Degeneracy}

Below we show that the degeneracy between the pointsource and finite-source solutions is resolved in favor of the point-lens solution. In addition to the slightly better fit to the data, it is found that the point-source solution is also supported by the external information obtained from astrometric and spectroscopic observations.

One line of evidence for the preference of the point-source solution comes from the identification of the blend with either the lens or its companion. This is identified from the astrometric measurement of the offset between the source and baseline object in the KMTNet images. From this measurement, it is found that the offset is 0.07 pixels, which corresponds to $\sim 28$ mas. The uncertainty in the position of the source measured on the difference image is $\sim 0.1$ pixel, and thus the measured astrometric offset is consistent with the measurement uncertainty of the baseline object on the template. The probability of a random field star with a brightness similar to or brighter than that of the blend with $V_{\mathrm{b}}=18.21$ lying this close to the source is extremely low, $p=3.6 \times 10^{-5}$. This indicates that the blend is not a field star that happens to lie close to the source. Excluding the possibility of a random field star, the blend can only be the lens itself, a companion to the lens, or a companion to the source. However, the blend cannot be a companion to the source because its spectroscopic type and observed color and magnitude place it well in the foreground. More specifically, being just slightly hotter than the Sun, with marginally higher surface gravity, the blend has $(V-I)_{0} \sim 0.68 \quad$ and $\quad M_{I} \sim 4.1 . \quad$ Hence, $E(V-I)=(V-I)_{\mathrm{b}}-(V-I)_{0, \mathrm{~b}}=1.08-0.68=0.4$ and so $A_{I} \simeq 1.25 E(V-I)=0.5$. These values imply a distance modulus $\mathrm{DM}=I_{\mathrm{L}}-M_{I, \mathrm{~L}}-A_{I, \mathrm{~L}} \simeq 12.5$, and thus the blend is located at a distance

$$
D_{\mathrm{b}}=10^{(\mathrm{DM}+5) / 5-3}=3.2 \pm 0.5 \mathrm{kpc} .
$$

We now argue that the blend is very likely to be the lens itself rather than its companion. The first point is that Equation (14) implies that the lens-source relative parallax is

$$
\pi_{\text {rel }}=\mathrm{au}\left(\frac{1}{D_{\mathrm{L}}}-\frac{1}{D_{\mathrm{S}}}\right)=0.188 \pm 0.042 \text { mas. }
$$

Here we adopt the distance to the source star of $D_{\mathrm{S}}=8 \mathrm{kpc}$. This is also the "blend-source relative parallax." Hence we can define an "Einstein radius of the blend"

$$
\theta_{\mathrm{E}, \mathrm{b}}=\left(\kappa M_{\mathrm{b}} \pi_{\mathrm{rel}}\right)^{1 / 2}=1.24 \pm 0.15 \text { mas, }
$$

where $M_{\mathrm{b}}$ denotes the mass of the blend. If the lens is the blend, then $\theta_{\mathrm{E}, \mathrm{b}}=\theta_{\mathrm{E}}$. But if not, then $\theta_{\mathrm{E}, \mathrm{b}}$ is still a useful concept. Now, suppose that the blend is a companion to the lens. We know that the mass ratio $Q \equiv M_{\mathrm{b}} / M_{1} \gg 1$ because the spectrum does not show significant light from a second star. Suppose that the lens and blend are separated by $\Delta \theta$. We define $S \equiv \Delta \theta / \theta_{\mathrm{E}}$, where again, $\theta_{\mathrm{E}}$ is the Einstein radius of the lens. Then the semi-diameter of the Chang-Refsdal (CR) caustic (in units of $\theta_{\mathrm{E}}$ ) is $w=2 Q / S^{2}$. We know that strictly $w<u_{0}=0.04$, because otherwise the light curve in Figure 1 would show huge residuals near the peak caused by the CR caustic. We estimate $w<u_{0} / 2$ to avoid detectable residuals. This leads to a limit

$$
\Delta \theta_{\min }=S_{\min } \theta_{\mathrm{E}}=\sqrt{\frac{4 Q \theta_{\mathrm{E}}^{2}}{u_{0}}}=\sqrt{\frac{4}{u_{0}}} \theta_{\mathrm{E}, \mathrm{b}} \sim 12.4 \mathrm{mas},
$$

because $\theta_{\mathrm{E}, \mathrm{b}}=\sqrt{Q} \theta_{\mathrm{E}}$. We note that $\Delta \theta_{\max }$ is independent of the lens mass $M_{1}$. But we also know that $\Delta \theta \lesssim 28$ mas, which is the measured astrometric offset between the source and baseline object. Hence, the allowed range in separation is about $1 / 2$ dex, which corresponds to $3 / 4$ dex in period, which is centered on roughly $\log (P /$ day $) \sim 5$.2. From Duquennoy \& Mayor (1991), about 6\% of G dwarfs have companions in this separation range. Again using Duquennoy \& Mayor (1991) statistics, the mean Einstein radius (hence cross section) of these companions will be lower than the primary by a factor of 0.64 . Hence, there is only a $0.64 \times 6 \% \simeq 4 \%$ chance that the lens is a companion to the blend rather than the blend itself. Finally, we consider the specific case of the "finite source" solutions, for which $\theta_{\mathrm{E}}=0.28$ mas (Equation (5)). Because $\theta_{\mathrm{E}} \ll \theta_{\mathrm{E}, \mathrm{b}}=\sqrt{Q} \theta_{\mathrm{E}}$, the lensing object responsible for the event is the lower-mass component of the binary with $Q=\left(\theta_{\mathrm{E}, \mathrm{b}} / \theta_{\mathrm{E}}\right)^{2}=(1.24 \mathrm{mas} / 0.28 \mathrm{mas})^{2} \sim 20$. As we just argued, the general probability that the lens is such a companion is low ( $5 \%)$. In addition, this would be a very unusual three-body system, i.e., a solar-mass star, a browndwarf companion at 50-100 au, orbited by a two-Neptunemass "moon" at about 1 au. Given that this model is already seriously disfavored by the microlensing data, we regard the low statistical probability just reported as well as the extreme nature of the system implied as essentially ruling out this possibility. Then, the only remaining possibility is that the blend is the lens itself.

Knowing that the blend is very likely to be the lens, another line of evidence supporting the point-source solution comes from the consistency of the external distance measurement of the lens, i.e., blend, by spectrum with the lensing parameters of the point-source solution. With the angular Einstein radius of $\theta_{\mathrm{E}} \simeq 1.24$ mas (Equation (16)) together with the estimated source radius of $\theta_{*} \simeq 0.28 \mu$ as (Equation (4)), the normalized source radius is

$$
\rho=\frac{\theta_{*}}{\theta_{\mathrm{E}}} \sim 2.3 \times 10^{-4} .
$$

The estimated value of $\rho$ is consistent with the centroid of the cloud of MCMC points in the $\Delta \chi^{2}$ distribution on the $q-\rho$ plane for the point-source solution presented in Figure 7. This indicates that the spectroscopically estimated lens distance is consistent with the lensing parameters of the point-source solution. In contrast, the spectroscopically estimated value of $\rho$ (Equation (18)) is significantly different from the value of the finite-source solutions, which is located in the range 
Table 3

Lens Parameters from Spectrum

\begin{tabular}{lcc}
\hline \hline \multicolumn{1}{c}{ Parameter } & Close & Wide \\
\hline$\theta_{\mathrm{E}}(\mathrm{mas})$ & $1.24 \pm 0.15$ & $\ldots$ \\
$\mu\left(\mathrm{mas} \mathrm{yr}^{-1}\right)$ & $7.0 \pm 0.9$ & $6.5 \pm 0.8$ \\
$M_{\mathrm{host}}\left(M_{\odot}\right)$ & $1.0 \pm 0.1$ & $\ldots$ \\
$M_{\mathrm{p}}\left(M_{\mathrm{J}}\right)$ & $4.5 \pm 0.6$ & $4.8 \pm 0.6$ \\
$D_{\mathrm{L}}(\mathrm{kpc})$ & $3.2 \pm 0.5$ & \\
$a_{\perp}(\mathrm{au})$ & $3.4 \pm 0.2$ & $5.0 \pm 0.3$ \\
$a(\mathrm{au})$ & $4.2 \pm 0.6$ & $6.1 \pm 0.6$ \\
$P(\mathrm{yr})$ & $8.6 \pm 1.9$ & $15.0 \pm 3.2$ \\
$v \sin i\left(\mathrm{~m} \mathrm{~s}^{-1}\right)$ & $(62 \pm 11) \sin i$ & $(55 \pm 10) \sin i$ \\
$I_{\mathrm{L}}(\mathrm{mag})$ & $17.13 \pm 0.01$ & $\ldots$ \\
$V_{\mathrm{L}}(\mathrm{mag})$ & $18.21 \pm 0.11$ & $\cdots$ \\
\hline
\end{tabular}

Note. $\theta_{\mathrm{E}}$ and $\mu$ denote the angular Einstein radius and the relative lens-source motion, respectively. $a_{\perp}$ and $a$ represent the projected planet-host separation at the time of the peak lensing magnification and the semimajor axis, respectively. $P$ denotes the orbital period and $v \sin i$ represents the RV amplitude. $I_{\mathrm{L}}$ and $V_{\mathrm{L}}$ represent the $I$ - and $V$-band magnitudes of the lens, respectively.

$8.5 \times 10^{-4} \lesssim \rho \lesssim 9.9 \times 10^{-4}$. We note that OGLE-2018BLG-0740 is the first external mass measurement of a microlens by spectrum, and this result is consistent with all microlens model information.

Although consistent, the physical lens parameters estimated from the spectrum are slightly different from those estimated from the Bayesian analysis. Therefore, we additionally list the lens parameters based on the spectrum in Table 3 .

\section{Radial-velocity Follow-up Observation}

The facts that (1) the blend is the lens, (2) the lens, with $I_{\mathrm{L}} \simeq 17.1$ and $V_{\mathrm{L}} \simeq 18.2$, is substantially brighter than typical lenses, and (3) its flux dominates the observed flux suggest that extra information such as the period and eccentricity of the planetary system can be additionally obtained from follow-up RV observations. In this section, we estimate the expected RV amplitude of the planetary system for future follow-up observations.

With the spectroscopically determined mass of the planet host, i.e., $M \sim 1.0 M_{\odot}$, together with the planet/host mass ratios of the point-source solutions, the mass of the planet is

$$
M_{\mathrm{p}}=q M_{\mathrm{host}}=\left\{\begin{array}{l}
4.5 \pm 0.6 M_{\mathrm{J}} \quad(\text { close }) \\
4.8 \pm 0.6 M_{\mathrm{J}} \quad(\text { wide })
\end{array}\right.
$$

where the upper and lower cases represent the values corresponding to the close and wide solutions, respectively. The projected host-planet separation is

$$
a_{\perp}=s \theta_{\mathrm{E}} D_{\mathrm{L}}=\left\{\begin{array}{l}
3.4 \pm 0.2 \text { au (close) } \\
5.0 \pm 0.3 \text { au (wide) }
\end{array}\right.
$$

Assuming a circular orbit and a random orientation of the planet around the host, the mean value of the intrinsic semimajor axis is

$$
\langle a\rangle=\sqrt{\frac{3}{2}} a_{\perp}=\left\{\begin{array}{lll}
4.2 \pm 0.6 \text { au } & \text { (close) } \\
6.1 \pm 0.6 \text { au } & \text { (wide) }
\end{array}\right.
$$

Table 4

Annual Parallax and Proper Motion

\begin{tabular}{lr}
\hline \hline Parameter & \multicolumn{1}{c}{ Value } \\
\hline$\pi(\mathrm{mas})$ & $0.27 \pm 0.19$ \\
$\mu_{E}\left(\operatorname{mas~yr}^{-1}\right)$ & $1.67 \pm 0.36$ \\
$\mu_{N}\left(\operatorname{mas~yr}^{-1}\right)$ & $-4.85 \pm 0.31$ \\
\hline
\end{tabular}

Note. The values are adopted from the Gaia archive. The quantity $\pi$ denotes the annual parallax, and $\mu_{E}$ and $\mu_{N}$ represent the east and north components of the proper motion, respectively.

From Kepler's third law, the orbital period of the planet is

$$
P=\left(\frac{a^{3}}{M}\right)^{1 / 2} \sim \begin{cases}8.6 \pm 1.9 \mathrm{yr} & \text { (close) }, \\ 15.0 \pm 3.2 \mathrm{yr} & \text { (wide) }\end{cases}
$$

Then, the expected RV amplitude is

$$
\begin{aligned}
& v \sin i=q\left(\frac{2 \pi a}{P}\right) \sin i \\
& \sim\left\{\begin{array}{l}
(62 \pm 11) \sin i \mathrm{~m} \mathrm{~s}^{-1} \quad \text { (close), } \\
(55 \pm 10) \sin i \mathrm{~m} \mathrm{~s}^{-1} \quad \text { (wide). }
\end{array}\right.
\end{aligned}
$$

These RV amplitudes are big enough to be measured using high-resolution spectrometers mounted on very large telescopes. For example, for a G-type star at $V=18$, Very Large Telescope (VLT)/Espresso can achieve $10 \mathrm{~m} \mathrm{~s}^{-1}$ precision with a single VLT telescope and $5-10 \mathrm{~m} \mathrm{~s}^{-1}$ precision by employing all four VLT telescopes. It should be noted that the $\mathrm{RV}$ signal, i.e., $v \sin i$, depends on the inclination of the planet orbit. For detections, therefore, the planet would need to have a large inclination, as pointed out by Clanton \& Gaudi (2014).

The motion of the lens is defined from the combination of spectroscopic and astrometric data. The radial velocity is measured from the Magellan spectrum as $v_{r}=-36 \pm 5 \mathrm{~km} \mathrm{~s}^{-1}$ after heliocentric correction. The projected velocity is estimated from the proper motion and distance by $\boldsymbol{v}=\boldsymbol{\mu} D_{\mathrm{L}}$. In Table 4 , we list the proper motion, $\boldsymbol{\mu}=\left(\mu_{E}, \mu_{N}\right)$, and annual parallax, $\pi$, of the lens from the list of Gaia data release 2 (Gaia DR2: Gaia Collaboration et al. 2018). Then, the east and north components of the projected lens velocity are $\left(v_{E}, v_{N}\right)=\left(\mu_{E}, \quad \mu_{N}\right) \quad D_{\mathrm{L}}=(25 \pm 5$, $-73 \pm 5) \mathrm{km} \mathrm{s}^{-1}$. We note that the distance to the lens, $2.2 \mathrm{kpc} \leqslant D_{\mathrm{L}} \equiv 1 / \pi \leqslant 12.5 \mathrm{kpc}$, estimated from the Gaia annual parallax, i.e., $\pi=0.27 \pm 0.19$ mas, is consistent with the spectroscopic measurement of $D_{\mathrm{L}}=3.2 \pm 0.5 \mathrm{kpc}$, but the uncertainty is very large due to the significant uncertainty of $\pi$.

Spectroscopic follow-up observations are important for two major scientific reasons. First, these observations would allow one to measure the period and eccentricity of the planet, which has not been done before for any microlens planet. Second, one can also probe for close-in planets, to which the RV method is sensitive. We note that the predicted period is long, $8.6 \mathrm{yr}$ for the close solution and $15.0 \mathrm{yr}$ for the wide solution, and thus the microlensing planet can be confirmed from spectroscopic follow-up observations that are conducted several times per year. We also note that denser sampling may enable the discovery of close-in rocky planets and will allow the first exploration of the planetary system architecture by combining the RV and microlensing methods. With these detections, the planetary system would be the closest analog of the solar 


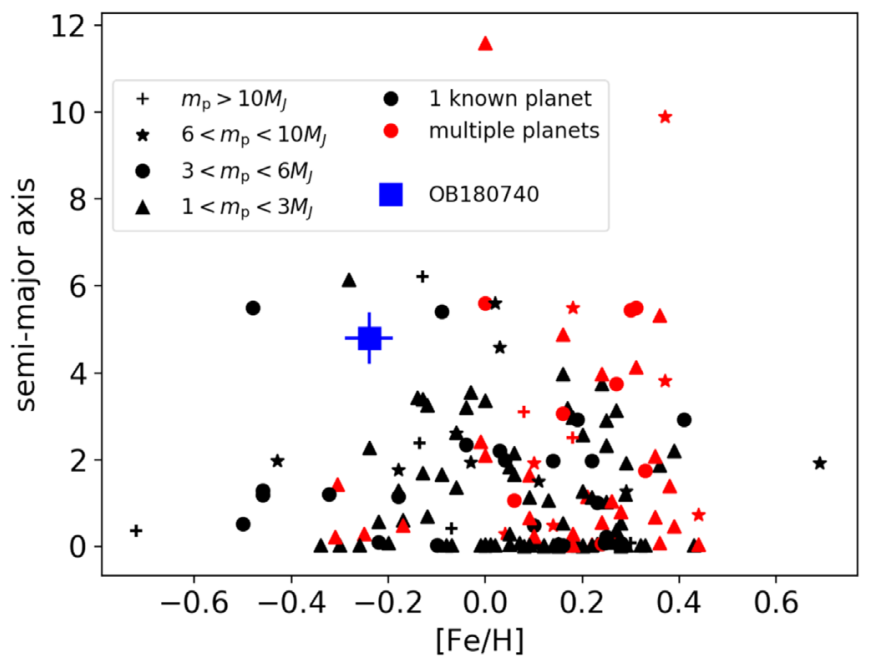

Figure 11. Comparison of OGLE-2018-BLG-0740Lb with the host star metallicities and semimajor axes of other exoplanets orbiting solar-type stars. Exoplanet data from the NASA Exoplanet Archive (downloaded 2019 April 11).

system ever, with a Sun-like star, a giant planet at the same distance as Jupiter, and close-in rocky planets.

Considering that the blend is very likely to be the host of the planet, the estimated metallicity of the host star of $[\mathrm{Fe} /$ $\mathrm{H}]=-0.24$ (Section 4) indicates that planet is a super-Jupiter orbiting a metal-poor host star. While there is a well-known correlation between giant planet frequency and host star metallicity, about $3 \%$ of metal-poor stars host giant planets (Gonzalez 1997; Santos et al. 2004; Fischer \& Valenti 2005). Figure 11 compares OGLE-2018-BLG-0740Lb with other known giant exoplanets $\left(1 M_{\mathrm{J}} \leqslant M_{\mathrm{p}} \leqslant 13 M_{\mathrm{J}}\right)$ orbiting solar-type stars $\left(0.8 M_{\odot} \leqslant M \leqslant 1.1 M_{\odot}\right)$. The discovered microlensing planet is located in a relatively underpopulated portion of exoplanet parameter space (large, distant planets orbiting lowmetallicity stars), but is similar to planets reported in Santos et al. (2010), Marmier et al. (2013), and Teske et al. (2016), which are all in systems with only one known planet.

\section{Conclusion}

We presented the analysis of the microlensing event OGLE2018-BLG-0740, which exhibited a strong short-term anomaly in the lensing light curve. We tested various interpretations of the anomaly and found that the event was produced by a planetary system. Despite the very strong signal, however, interpreting the anomaly suffered from two types of degeneracies, in which one was caused by the previously known close/wide degeneracy, while the other degeneracy was caused by the ambiguity in the normalized source radius, finite/pointsource degeneracy, due to the incomplete coverage of the anomaly. With the external information obtained from astrometric and spectroscopic observations, we identified that the lens was the blend and this led to the resolution of the finite/ point-source degeneracy in strong favor of the point-source solution. It was found that the lens was a planetary system composed of a super-Jupiter planet around a solar-mass star located at a distance of $\sim 3 \mathrm{kpc}$. The bright nature of the lens combined with its dominance of the observed flux suggested that the period and eccentricity of the microlensing planet could be measured for the first time via RV observations using a high-resolution spectrometers mounted on large telescopes. We presented the expected RV amplitude for future spectroscopic observation.

Work by C.H. was supported by the grant (2017R1A4A1015178) of National Research Foundation of Korea. Work by A.G. was supported by US NSF grant AST1516842. Work by I.G.S. and A.G. were supported by JPL grant 1500811. A.G. received support from the European Research Council under the European Union's Seventh Framework Programme (FP 7) ERC grant Agreement No. [321035]. This research has made use of the NASA Exoplanet Archive, which is operated by the California Institute of Technology, under contract with the National Aeronautics and Space Administration under the Exoplanet Exploration Program. S.D. acknowledges Project 11573003 supported by National Science Foundation of China (NSFC). The MOA project is supported by JSPS KAKENHI grant Nos. JSPS24253004, JSPS26247023, JSPS23340064, JSPS15H00781, JP16H06287, and JP17H02871. Y.M. acknowledges the support of the grant JP14002006. D.P.B., A.B., and C.R. were supported by NASA through grant NASA-80NSSC18K0274. The work by C.R. was supported by an appointment to the NASA Postdoctoral Program at the Goddard Space Flight Center, administered by USRA through a contract with NASA. N.J.R. is a Royal Society of New Zealand Rutherford Discovery Fellow. The OGLE project has received funding from the National Science Centre, Poland, grant MAESTRO 2014/14/A/ST9/00121 to A.U. This research has made use of the KMTNet system operated by the Korea Astronomy and Space Science Institute (KASI) and the data were obtained at three host sites of CTIO in Chile, SAAO in South Africa, and SSO in Australia. We acknowledge the high-speed internet service (KREONET) provided by Korea Institute of Science and Technology Information (KISTI). We acknowledge the spectral fitting done by Yang Huang and Huawei Zhang.

\section{ORCID iDs}

Cheongho Han (ib https://orcid.org/0000-0002-2641-9964 Valerio Bozza (iD https://orcid.org/0000-0003-4590-0136 Subo Dong (1D https://orcid.org/0000-0002-1027-0990 Konstantina Boutsia (ib https://orcid.org/0000-00034432-5037

Michael D. Albrow (iD https://orcid.org/0000-0003-3316-4012 Sun-Ju Chung (iD https://orcid.org/0000-0001-6285-4528 Kyu-Ha Hwang (i) https://orcid.org/0000-0002-9241-4117 Kyeongsoo Hong (ib https://orcid.org/0000-0002-8692-2588 Jan Skowron (iD https://orcid.org/0000-0002-2335-1730 Paweł Pietrukowicz (iD https://orcid.org/0000-0002-2339-5899 Krzysztof Ulaczyk (i) https://orcid.org/0000-0001-6364-408X Patryk Iwanek (iD https://orcid.org/0000-0002-6212-7221 David P. Bennett (iD https://orcid.org/0000-0001-8043-8413 Akihiko Fukui (1) https://orcid.org/0000-0002-4909-5763 Yoshitaka Itow (iD https://orcid.org/0000-0002-8198-1968 Naoki Koshimoto (iD https://orcid.org/0000-0003-2302-9562 Shota Miyazaki (iD https://orcid.org/0000-0001-9818-1513 Nicholas J. Rattenbury (D) https://orcid.org/0000-00015069-319X

Daisuke Suzuki (iD https://orcid.org/0000-0002-5843-9433 


\section{References}

Alard, C., \& Lupton, R. H. 1998, ApJ, 503, 325

Albrow, M. 2017, MichaelDAlbrow/pyDIA: Initial Release on Github, Zenodo, doi:10.5281/zenodo.268049

Albrow, M. D., Horne, K., Bramich, D. M., et al. 2009, MNRAS, 397, 2099 An, J. H. 2005, MNRAS, 356, 1409

Bensby, T., Adén, D., Meléndez, J., et al. 2011, A\&A, 533, 134

Bessell, M. S., \& Brett, J. M. 1988, PASP, 100, 1134

Bond, I. A., Abe, F., Dodd, R. J., et al. 2001, MNRAS, 327, 868

Chabrier, G. 2003, ApJL, 586, L133

Chung, S.-J., Han, C., Park, B.-G., et al. 2005, ApJ, 630, 535

Clanton, C., \& Gaudi, B. S. 2014, ApJ, 791, 90

Dominik, M. 1998, A\&A, 329, 361

Dominik, M. 1999, A\&A, 349, 108

Dominik, M., Bachelet, E., Bozza, V., et al. 2019, MNRAS, 484, 5608

Dressler, A., Bigelow, B., Hare, T., et al. 2011, PASP, 123, 288

Duquennoy, A., \& Mayor, M. 1991, A\&A, 248, 485

Fischer, D. A., \& Valenti, J. 2005, ApJ, 622, 1102

Gaia Collaboration et al. 2018, A\&A, 616, 1

Gaudi, B. S. 1998, ApJ, 506, 533

Gaudi, B. S., \& Gould, A. 1997, ApJ, 486, 85

Gonzalez, G. 1997, MNRAS, 285, 403

Gonzalez, O. A., Rejkuba, M., Zoccali, M., et al. 2012, A\&A, 543, 13

Goodman, J., \& Weare, J. 2010, CAMCOS, 5, 65

Gould, A. 1992, ApJ, 392, 442

Gould, A. 1997, in Variables Stars and the Astrophysical Returns of the Microlensing Surveys, ed. R. Ferlet, J.-P. Maillard, \& B. Raban (Gif-surYvette: Editions Frontieres), 125

Gould, A. 2000, ApJ, 535, 928

Gould, A., \& Loeb, A. 1992, ApJ, 396, 104
Griest, K., \& Safazadeh, N. 1998, ApJ, 500, 37

Han, C. 2006, ApJ, 638, 1080

Han, C., Bond, I. A., Gould, A., et al. 2018a, AJ, 156, 226

Han, C., \& Gould, A. 1995, ApJ, 447, 53

Han, C., \& Gould, A. 2003, ApJ, 592, 172

Han, C., Jung, Y. K., Udalski, A., et al. 2018b, ApJ, 867, 136

Hwang, K.-H., Udalski, A., Shvartzvald, Y., et al. 2018, AJ, 155, 20

Jung, Y. K., Udalski, A., Yee, J. C., et al. 2017, AJ, 153, 129

Kervella, P., Thévenin, F., Di Folco, E., \& Ségransan, D. 2004, A\&A, 426, 29

Kim, D.-J., Kim, H.-W., Hwang, K.-H., et al. 2018, AJ, 155, 76

Kim, S.-L., Lee, C.-U., Park, B.-G., et al. 2016, JKAS, 49, 37

Koleva, M., Prugniel, Ph., Bouchard, A., \& Wu, Y. 2009, A\&A, 501, 1269

Mao, S., \& Paczyński, B. 1991, ApJL, 374, L37

Marmier, M., Ségransan, D., Udry, S., et al. 2013, A\&A, 551, A90

Moultaka, J., Ilovaisky, S. A., Prugniel, P., \& Soubiran, C. 2004, PASP, 116, 693

Nataf, D. M., Gould, A., Fouqué, P., et al. 2013, ApJ, 769, 88

Santos, N. C., Israelian, G., \& Mayor, M. 2004, A\&A, 415, 1153

Santos, N. C., Mayor, M., Benz, W., et al. 2010, A\&A, 512, A47

Shin, I.-G., Yee, J. C., Gould, A., et al. 2019, arXiv:1902.10945

Skowron, J., Ryu, Y.-H., Hwang, K.-H., et al. 2018, AcA, 68, 43

Sumi, T., Abe, F., Bond, I. A., et al. 2003, ApJ, 591, 20

Szymański, M. K., Udalski, A., Soszyński, I., et al. 2011, AcA, 61, 83

Teske, J. K., Shectman, S. A., Vogt, S. S., et al. 2016, AJ, 152, 167

Udalski, A. 2003, AcA, 53, 291

Udalski, A., Szymański, M. K., \& Szymański, G. 2015, AcA, 65, 1

Wu, Y., Singh, H. P., Prugniel, P., Gupta, R., \& Koleva, M. 2011, A\&A, 525,71

Yee, J. C., Shvartzvald, Y., Gal-Yam, A., et al. 2012, ApJ, 755, 102

Yoo, J., DePoy, D. L., Gal-Yam, A., et al. 2004, ApJ, 603, 139 